\title{
Effects of auricular acupuncture on appetite in patients with advanced cancer: a pilot randomized controlled trial
}

\author{
Lingyun Sun ${ }^{1}$, Jun J. Mao ${ }^{2}$, Qun Liu ${ }^{3}$, Yufei Yang ${ }^{1}$, Bin $\mathrm{He}^{1}$ \\ ${ }^{1}$ Clinical Cancer Center, Xiyuan Hospital, China Academy of Chinese Medical Sciences, Beijing 100091, China; ${ }^{2}$ Memorial Sloan Kettering Cancer \\ Center, Bendheim Integrative Medicine Center, New York, NY, USA; ${ }^{3}$ Department of Integrative Chinese and Western Medicine, Cancer Hospital \\ of Peking University, Beijing 100142, China \\ Contributions: (I) Conception and design: B He, J Mao, L Sun; (II) Administrative support: Y Yang, B He; (III) Provision of study materials or \\ patients: B He; (IV) Collection and assembly of data: Q Liu; (V) Data analysis and interpretation: L Sun; (VI) Manuscript writing: All authors; (VII) \\ Final approval of manuscript: All authors. \\ Correspondence to: Bin He, MD. Clinical Cancer Center, Xiyuan Hospital, China Academy of Chinese Medical Sciences, \#1 Xiyuan Caochang Road, \\ Haidian District, Beijing 100091, China. Email: hebinbj68@163.com.
}

Background: Over half of patients with advanced cancer report appetite loss or anorexia. Previous studies have shown the benefit of acupuncture for cancer-related nausea and vomiting, but limited evidence exists for its role in appetite improvement. Our study aimed to evaluate the feasibility and safety of auricular acupuncture to improve appetite for cancer patients with advanced disease.

Methods: We performed a two-arm parallel, pilot randomized controlled trial (RCT) of auricular acupuncture versus usual care control in patients with stage III or IV cancer who experienced appetite loss. The primary outcome was changed in the Simplified Nutritional Appetite Questionnaire (SNAQ; score range, 4-20) between two groups from baseline to weeks 2 and 4, with secondary outcomes including change in weight, as well as an additional evaluation at week 8 for durability of treatment effects. We used independent two-sample $t$-test for the change in mean score for each outcome during or after treatment. We assessed the interaction between time and treatment from baseline to weeks 2, 4, and 8 using mixed-effects models by ANOVA test.

Results: We randomized 55 patients to auricular acupuncture $(\mathrm{N}=27)$ or control group $(\mathrm{N}=28)$. By week 4, the auricular acupuncture group had a significantly higher escalation in the SNAQ score than the control group compared with baseline [mean difference 3.69; 95\% confidential interval (95\% CI): 2.5, 4.8; $\mathrm{P}<0.001$ ] and experienced a $51.4 \%$ improvement in appetite. From baseline to weeks 2 and 4, patients lost a little weight in the control group but gained weight in the auricular acupuncture group. Between baseline and week 4, change in the SNAQ score was significantly associated with change in weight $(\mathrm{P}=0.001)$. No adverse events (AEs) were reported during the study.

Conclusions: Compared to usual care, auricular acupuncture is feasible and safe to improve appetite and help patients with advanced cancer gain weight.

Keywords: Auricular acupuncture; advanced cancer; anorexia; expectancy; randomized controlled trial (RCT)

Submitted Jan 19, 2020. Accepted for publication Mar 02, 2020.

doi: $10.21037 /$ apm.2020.04.24

View this article at: http://dx.doi.org/10.21037/apm.2020.04.24 


\section{Introduction}

Over half of cancer patients with advanced disease experience appetite loss or anorexia, which has significant relevance in predicting weight loss and development of cachexia $(1,2)$. Despite the negative prognostic role of appetite loss in patients' quality of life and survival outcomes, there is still a lack of effective treatments $(3,4)$. Pharmacologic therapies such as melatonin and anamorelin may help cancer patients regain their appetite $(5,6)$. However, those drugs can increase the frequency of nausea, and the risk of hyperglycemia and embolism (7). Emerging efforts are focused on evaluating non-pharmacologic therapies such as exercise and psychosocial support to limit appetite loss or cachexia in cancer patients $(8,9)$.

Acupuncture, an important part of complementary and alternative medicine (CAM), has been used to alleviate chemo-induced or cancer-related nausea and vomiting $(10,11)$. Studies indicate that acupuncture interventions may be associated with the increased secretion of ghrelin and cholecystokinin and decreased 5-HT secretion (10). These mechanisms could also partly explain how cancer patients with advanced disease develop anorexia or cachexia (12). Given these facts, some feasibility studies have been conducted to evaluate acupuncture's effect on treating anorexia among cancer patients with advanced disease. Patients from these studies showed some improvement in appetite (13).

Auricular acupuncture is a novel type of acupuncture, in which an acupuncturist stimulates acupoints on the ears using small needles or nodules (14). It is more convenient for outpatients, who may not have daily access to hospitals or clinics. In addition, patients or caregivers can press these points themselves, which makes the intervention more controllable and operable. In China, it is common practice for traditional Chinese medicine (TCM) hospitals to utilize auricular acupressure to reduce chemo-induced nausea and vomiting (15). An Australian qualitative study also showed that auricular acupuncture was welcomed by cancer patients who suffered from anorexia (16). However, to the best of our knowledge, there is still no reported clinical trial to evaluate the effect of auricular acupuncture's role in addressing this problem. We conducted a pilot randomized controlled trial (RCT) to evaluate the preliminary effect and safety of auricular acupuncture compared with usual care on appetite among cancer patients with advanced disease.

\section{Methods}

We conducted a two-arm parallel pilot RCT from May 2016 to December 2017 at Xiyuan Hospital of China Academy of Chinese Medical Sciences. The study was approved by Ethics Review Board of Xiyuan Hospital (No. 2016XLA115-1) and written informed consent was obtained from the patient for publication of this study and any accompanying images. The trial had been registered on ClinicalTrials.gov (NCT02887586, register date: September 2, 2016).

\section{Participants}

Eligible individuals were pathologically diagnosed cancer patients (any cancer type), with stage III or IV diseases (AJCC eighth edition). Patients need to meet cancerrelated appetite loss or anorexia diagnostic criteria which was measured by a symptom-based anorexia assessment form (including 12 questions and a total score lower than 24 indicating anorexia). Other inclusion criteria included age between 18 and 80 years and life expectancy over 3 months. Exclusion criteria included patients who had physiological eating disorders such as anorexia nervosa, bulimia nervosa, and binge eating disorder, as well as gastrointestinal obstruction, current use of steroid medication, and heart disease or diabetes. All participants consented before randomization.

\section{Randomization}

Participants were randomized into the auricular acupuncture or control group with a 1:1 ratio. A thirdparty statistician used computer-generated numbers (SAS 9.2) sealed in opaque envelopes. All participants were sequentially randomized into different group after screening and consent.

\section{Interventions}

In the treatment group, patients received auricular acupuncture in outpatient clinic. We would offer patients with usual nutritional supportive care such as oral nutrient, according to the China Anti-Cancer Association's (CACA) Cancer Anorexia Diagnose and Treatment Guidelines, if they need. Main ear-acupoints included shenmen, stomach, 


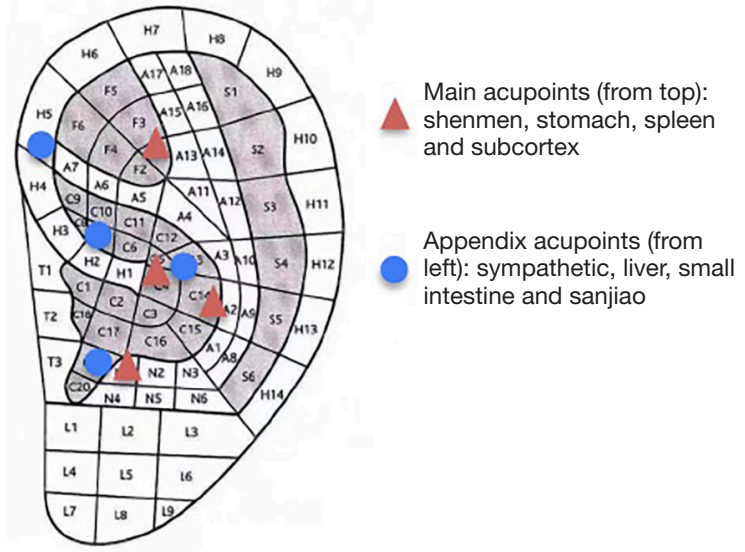

Figure 1 Main and appendix acupoints on standard model diagram. Red triangle: main acupoints (from top): shenmen, stomach, spleen, and subcortex; blue cycle: appendix acupoints (from left): sympathetic, liver, small intestine and sanjiao.

spleen and subcortex. Other appendix acupoints were sympathetic, liver, small intestine and sanjiao (Figure 1). The treating physician taped intradermal needles onto acupoints on one ear and pressed each point for about 2 minutes. Patients were instructed to press the needles two or three times per day by themselves. After 2 or 3 days, the physician removed and discarded the used needles and placed new needles onto the other ear. The treatment lasted for 4 weeks and we observed patients for another 4 weeks to assess the long-term durability of effects. Patients in the control group received usual nutritional supportive care and had the option to receive auricular acupuncture treatment after completing the 8-week observation period.

\section{Outcomes and follow-up}

The primary outcome was the average appetite score, as measured by the Simplified Nutritional Appetite Questionnaire (SNAQ), which is known to be reliable, valid, and responsive to treatment effects (17). The SNAQ includes four items assessing appetite, when one feels full, how good food tastes while eating, and daily food intake frequency. Each item is measured using a five-point Likert scale from a to e, (indicating a score of 1 to 5). A total SNAQ score of no more than 14 indicates significant risk of $5 \%$ weight loss within 6 months; a higher SNAQ score indicates a better appetite level.

The secondary outcome was patients' body weight. We measured body weight in kilograms ( $\mathrm{kg}$ ) using a standard weight scale and collected it at weeks 2 and 4 during treatments, and at the week 8 follow-up. We also used the Acupuncture Expectancy Scale at each visit to evaluate patients' expectancy about auricular acupuncture treatments (18). Research staff monitored adverse events (AEs) using a standard $\mathrm{AE}$ case report form each week during the intervention phase.

We collected background information such as age, gender, and body mass index (calculated variable through height and weight) at baseline. We also recorded participants' disease information including cancer type, stage, time since diagnosis, and Karnofsky Performance Status (KPS) according to their medical records.

\section{Statistical analysis}

Patients were considered evaluable for analysis if they provided both baseline and treatment assessments. We used an analysis of variance or chi-square test to compare baseline variables between groups. The predefined primary end point for the trial was the two-group comparison of mean change in SNAQ score from baseline to the middle and end of intervention (weeks 2 and 4 ) between the auricular and control groups by independent two-sample $t$-test. Changes in secondary outcomes, including weight and expectations of acupuncture were also compared between groups. We completed a correlation analysis between SNAQ score and weight at weeks 2 and 4 . We also used a multivariate model to evaluate the association between acupuncture expectancy score at baseline and SNAQ score at week 4, controlled by baseline SNAQ score and treatment group. Because primary and secondary outcome measures were repeated over time, differences in changes were assessed from baseline to weeks 2, 4 , and 8 using mixed effects models by ANOVA test. We did the same comparison at the follow-up visit at week 8 . Differences were considered statistically significant if the $\mathrm{P}$ value was lower than 0.05 . As a pilot trial focused on feasibility, we defined the sample size as 60 in consideration of $5 \%$ of the participants who may drop out and be lost to follow-up. All analyses were performed using SPSS 21 (IBM Corp., USA).

\section{Patient and public involvement}

During the design phase of the study, we had conducted pilot study among outpatients in our clinic and asked for their experiences of the intervention. Patients were 


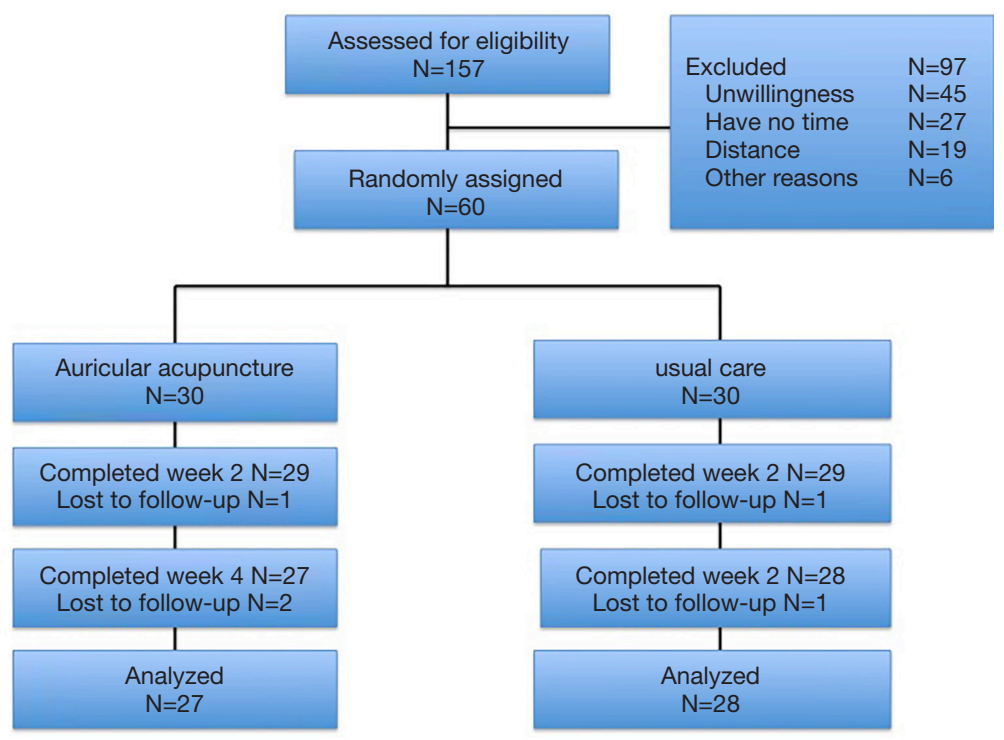

Figure 2 CONSORT diagram.

encouraged to refer other patients who experienced appetite loss to participate the study. We did not involve patients for conduct of the study. For all patients who participated the study, we will share our results to them during follow-up or through social media. As a usual care-controlled study, we consented and explained group assignment to all patients before randomization and we offered additional auricular acupuncture treatments to patients in usual care group after 8 weeks of observation. Our results also showed that expectations to acupuncture treatments did not impact the main outcomes for both groups.

\section{Results}

Between September 2016 and June 2017, we screened 157 patients (Figure 2); 97 (62\%) declined to participate because of unwillingness, distance, and limited time. Of the 60 randomized patients, two did not receive any treatment and three dropped out after at least one treatment. In total, 27 patients in the acupuncture group and 28 in the control group completed all visits.

\section{Baseline patient characteristics and disease information}

The mean age of these 55 participants was $65 \pm 11$ (range, 31 to 80 ) years old. Among them, $53 \%$ were female, $63 \%$ had stage IV disease, 38\% had gastrointestinal cancer, and $23 \%$ had lung cancer. There was no significant difference between the distribution of gender, age, stage, and tumor site. However, in the acupuncture group more patients had a KPS score less than or equal to $60(\mathrm{P}=0.04)$, indicating that the average health of patients in this group was worse than those in the control group (Table 1).

\section{Appetite improvement}

At baseline, the SNAQ scores for auricular acupuncture and control groups were $8.70 \pm 2.00$ and $9.5 \pm 1.85$, respectively, indicating that patients in both groups experienced poor appetite (a SNAQ score lower than 14 predicts a risk of $5 \%$ weight loss). By week 4 , the auricular acupuncture group had a significantly higher change in the SNAQ score than the control group compared with baseline [mean difference 3.69; $95 \%$ confidential interval (95\% CI): 2.5 , 4.8; $\mathrm{P}<0.001]$, with an escalation of 4.48 points in the auricular acupuncture group and a decline of 0.07 point in the control group. We observed differences in SNAQ score changes between groups during the intervention (from baseline to week 2 and $4, \mathrm{P}<0.001$ for time and treatment interaction by ANOVA measurement). Compared with baseline, participants in the auricular acupuncture group experienced a $51.4 \%$ improvement in appetite by week 4 (Figure 3 and Table 2). 
Table 1 Participant characteristics between auricular acupuncture and control group

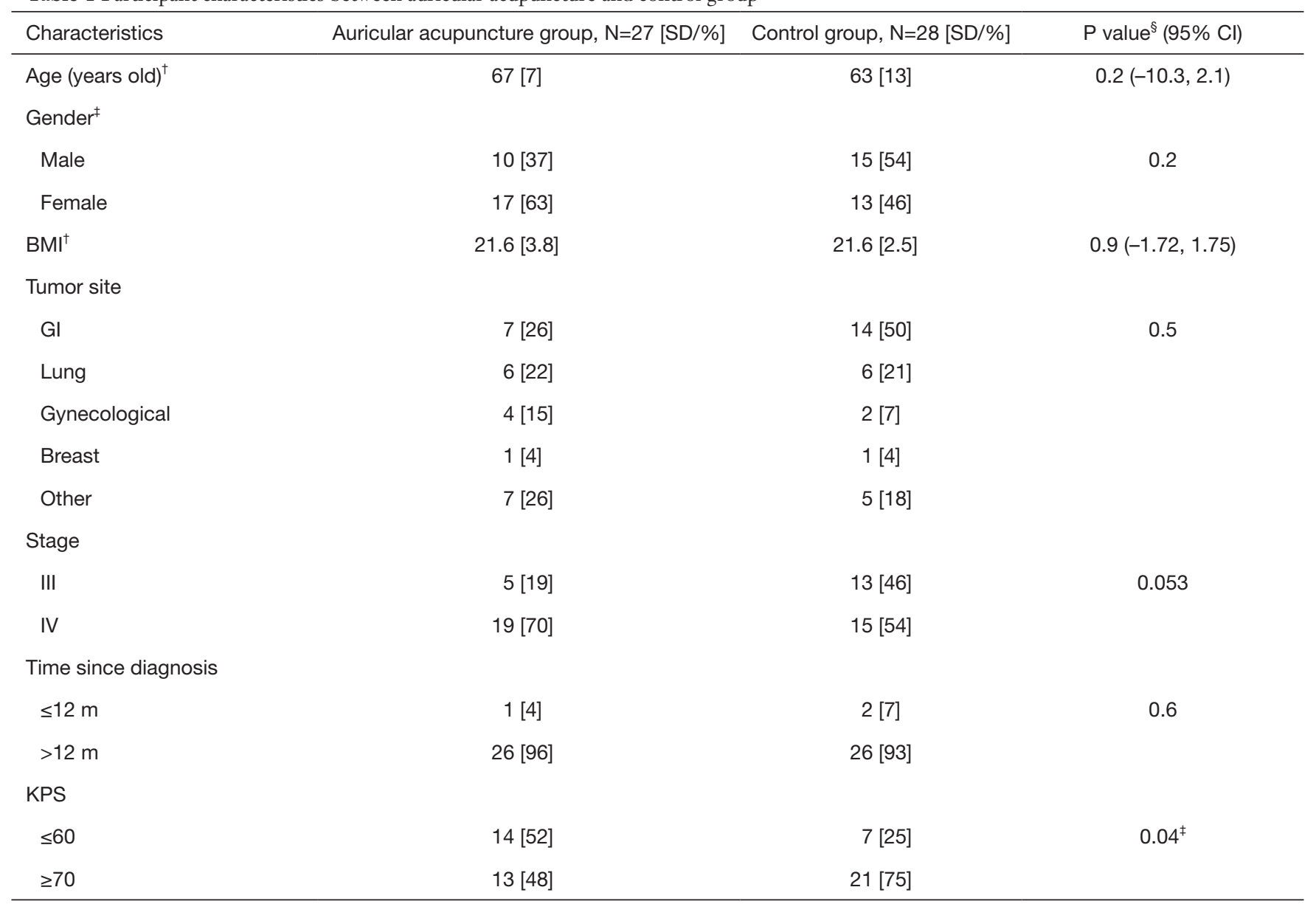

${ }^{\dagger}$, For continuous variables, including age and BMI, we used a $t$-test to compare the mean differences between groups; ${ }^{\ddagger}$, for categorized variables, including gender, tumor site, stage, time since diagnosis, and KPS, we used Fisher's exact test; ${ }^{\S}$, statistical significance was set at $\mathrm{P}=0.05$. SD, standard deviation; $\mathrm{Cl}$, confidential interval; GI, gastrointestinal (cancer); KPS, Karnofsky Performance Status.

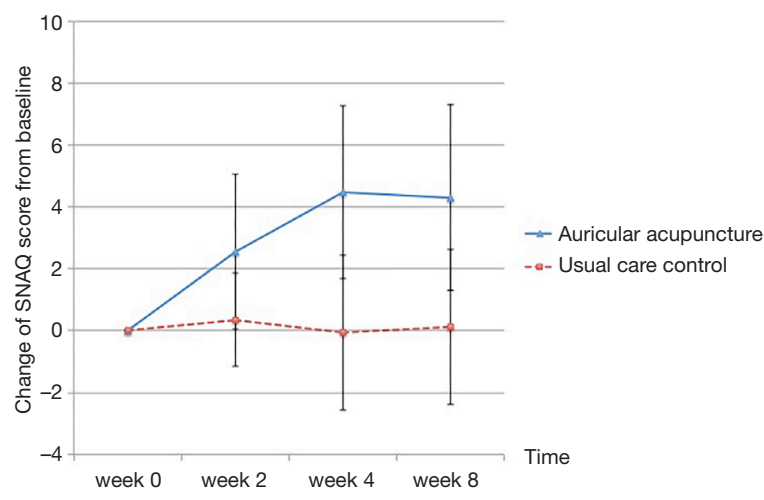

Figure 3 Change in SNAQ score between treatment groups at baseline, weeks 2 and 4 during treatments, and week 8 at followup. SNAQ, Simplified Nutritional Appetite Questionnaire.

\section{Secondary outcomes}

Trends showed that patients in the control group had lost a little weight, but that those in the auricular acupuncture group gained weight from baseline by week 2 and 4 (statistically significant by week 4, with $1.88 \mathrm{~kg}$ higher weight in the acupuncture group than in the control group; 95\% CI: 1.24, 2.54; P<0.001; Figure 4). Between baseline and week 4 , the change in the SNAQ score was significantly associated with a change in weight (Pearson Correlation Coefficient $0.43 ; \mathrm{P}=0.001$ ).

Patients' expectancy of acupuncture [0-4] gradually declined in the control group (from $10.0 \pm 4.4$ at baseline to $9.0 \pm 4.4$ at week $4 ; \mathrm{P}=0.4$ ), but increased in the acupuncture 
Table 2 Change in appetite (SNAQ score) by treatment group

\begin{tabular}{lccc}
\hline Appetite level (SNAQ score) & Auricular acupuncture $(\mathrm{n}=27)$ & Control group $(\mathrm{n}=28)$ & $\mathrm{P}_{\mathrm{value}}^{\dagger}$ \\
\hline Baseline SNAQ score & $8.70(2.00)$ & $9.57(1.85)$ & 0.1 \\
Change from baseline at week 2 & $2.56(2.50)$ & $0.36(1.47)$ & $<0.001^{\dagger}$ \\
Change from baseline at week 4 & $4.48(2.81)$ & $-0.07(2.52)$ & $<.11(2.47)$ \\
Change from baseline at week 8 & $4.30(3.00)$ & $<0.001^{\dagger}$ & $<0.001^{\dagger}$ \\
\hline
\end{tabular}

All values are mean (SD). ${ }^{\dagger}$, Statistical significance was set at $\mathrm{P}=0.05 . \mathrm{SNAQ}$, Simplified Nutritional Appetite Questionnaire; SD, standard deviation.

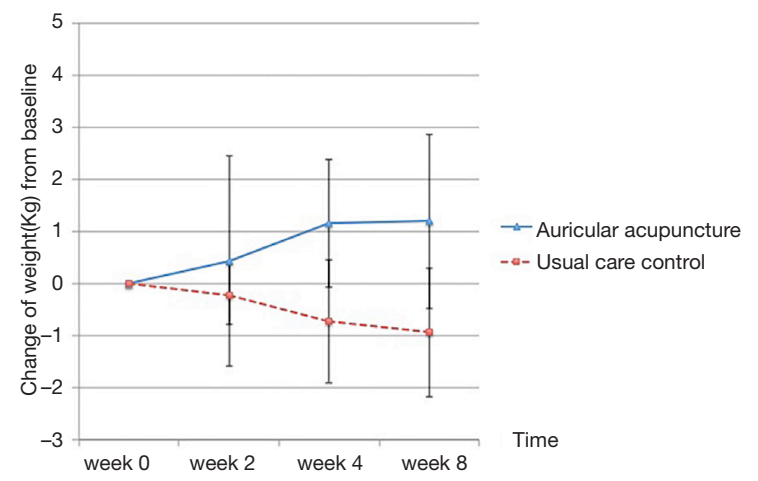

Figure 4 Patients' changes in body weight between groups at baseline, week 2 and 4 during treatments, and at the week 8 followup visit.

group (from $12.3 \pm 3.7$ to $14.3 \pm 5.1, \mathrm{P}=0.065$ ). Expectancy at baseline did not impact the main outcome at week 4 in the multivariate regression model (Coef. 0.036; $\mathrm{P}=0.5 ; 95 \% \mathrm{CI}$ : $-0.08,1.5)$.

\section{Durability of treatment effects}

By week 8 from random assignment (the fourth week after the intervention ended) patients in the acupuncture group still reported better appetite than the control group (change of SNAQ score from baseline $4.3 \pm 3.0 \mathrm{vs.} 0.11 \pm 2.47$; $\mathrm{P}<0.001$; Table 2). Compared with baseline, participants in the auricular acupuncture group gained on average $1.2 \mathrm{~kg}$ of weight at week 8 , but those in the control group lost $0.9 \mathrm{~kg}$ $(\mathrm{P}<0.001$, Figure 4).

\section{AEs}

There were no significant AEs in this study. Three patients reported tolerable pain on the ear related to the needle, especially when touching or pressing the needle.

\section{Discussion}

In this pilot RCT, we found that auricular acupuncture improved appetite and weight in patients with advanced cancer compared to usual care. The intervention may also have certain durability effects on appetite 4 weeks after the end of treatment. In addition, auricular acupuncture is safe for cancer patients to improve their appetite, as no significant AEs were reported.

A systematic review revealed that acupuncture and related therapies showed improvement in anorexia among cancer patients, but the difference was not statistically significant (total sample size $\mathrm{n}=50,95 \%$ CI: 0.94,6.72) (3). Only a few studies have been conducted to evaluate the feasibility of using auricular acupuncture to manage these symptoms $(16,19)$. Compared to previous studies, ours was an RCT with a larger sample size. Thus, our results provide stronger evidence for acupuncture, in particular for auricular acupuncture's role in alleviating appetite loss or anorexia and weight gain in cancer patients with advanced diseases. The durability effect of auricular acupuncture was consistent with observations in studies of fatigue and hot flashes $(20,21)$.

The endocrine manifestation mechanism could partly explain how auricular acupuncture may improve cancerrelated appetite loss. In animal research, auricular acupuncture improved food intake of rectal cancer model rats with significantly increased serum leptin and orexin-A (22). Leptin is an anorexigenic adipokine, which could stimulate secretion of GnRH and hyperleptinemia and may be associated with reduction of body fat mass (23). Orexin-A is a neuropeptide secreted by the lateral and posterior hypothalamic areas and plays an important role in feeding behavior regulation (24). Both appetite-regulating hormones indicate the potential neuropsychiatric mechanism of cancer-related appetite loss. A study from Shanghai showed that nearly $50 \%$ of 
advanced cancer patients with cachexia also suffered from distress and loss of energy (25). From the prospective view of TCM, acupuncture may affect patients' appetite by increasing their desire to eat, rather than increasing the physical feeling of hunger. Further qualitative research is necessary to discover more about the psycho-physical interaction of cancer-related appetite loss and acupuncture interventions (9).

Our study has certain limitations. As a pilot clinical trial, we did not use sham control and blinding methods, which may add observer bias. Since we did not offer patients in the control group active treatment, their appetite could be influenced by their psychological satisfaction. Our inclusion criteria did not restrict certain types of malignancies, which made subgroup analysis complex. According to our study, lung and GI cancer patients were more likely to experience cancer-related appetite loss. Future studies could focus on these cancer types and the differences in treatment effectiveness. In addition, researchers should conduct a longitudinal study on the long-term effect of auricular acupuncture to prevent cachexia development and benefit survival outcomes.

In conclusion, auricular acupuncture is effective and safe for improving appetite and helping cancer patients with advanced disease gain weight compared to usual care. This intervention also had a durable effect on appetite after the end of treatment. These preliminary findings need to be confirmed in larger studies with sham control and longterm follow-up for cachexia development and survival outcomes. For cancer patients with advanced diseases, auricular acupuncture is an operable, convenient, safe, and effective treatment to help them address the problem of appetite loss and acquire better quality of life.

\section{Acknowledgments}

We thank our student workers for their dedication to clinical trial coordination, data collection and management, and data input. Sincere thanks also go to the patients, patient advisers, oncologists, nurses, and clinical staff for their support of this study.

Funding: This study was funded by a grant from the China National Science Foundation Council (No. 81573958, 2015). It was also supported in part by a National Institutes of Health/National Cancer Institute Cancer Center grant (grant number P30 CA008748) and the Translational and Integrative Medicine Research Fund at Memorial Sloan Kettering Cancer Center (Dr. JJM).

\section{Footnote}

Conflicts of Interest: All authors have completed the ICMJE uniform disclosure form (available at http://dx.doi. org/10.21037/apm.2020.04.24). The authors have no conflicts of interest to declare.

Ethical Statement: The authors are accountable for all aspects of the work in ensuring that questions related to the accuracy or integrity of any part of the work are appropriately investigated and resolved. The study was conducted in accordance with the Declaration of Helsinki (as revised in 2013). The study was approved by Ethics Review Board of Xiyuan Hospital (No. 2016XLA1151) and written informed consent was obtained from the patient for publication of this study and any accompanying images. The trial had been registered on ClinicalTrials.gov (NCT02887586, register date: September 2, 2016).

Open Access Statement: This is an Open Access article distributed in accordance with the Creative Commons Attribution-NonCommercial-NoDerivs 4.0 International License (CC BY-NC-ND 4.0), which permits the noncommercial replication and distribution of the article with the strict proviso that no changes or edits are made and the original work is properly cited (including links to both the formal publication through the relevant DOI and the license). See: https://creativecommons.org/licenses/by-nc-nd/4.0/.

\section{References}

1. Vagnildhaug OM, Brunelli C, Hjermstad MJ, et al. A prospective study examining cachexia predictors in patients with incurable cancer. BMC Palliat Care 2019;18:46.

2. Bens A, Papadopoulos FC, Pukkala E, et al. Worse survival after breast cancer in women with anorexia nervosa. Breast Cancer Res Treat 2018;168:495-500.

3. Zhang F, Shen A, Jin Y, et al. The management strategies of cancer-associated anorexia: a critical appraisal of systematic reviews. BMC Complement Altern Med 2018;18:236.

4. Childs DS, Jatoi A. A hunger for hunger: a review of palliative therapies for cancer-associated anorexia. Ann Palliat Med 2019;8:50-8.

5. Ruiz-García V, López-Briz E, Carbonell-Sanchis R, et al. Megestrol acetate for cachexia-anorexia syndrome. A systematic review. J Cachexia Sarcopenia Muscle 2018;9:444-52. 
6. Hamauchi S, Furuse J, Takano T, et al. A multicenter, open-label, single-arm study of anamorelin (ONO-7643) in advanced gastrointestinal cancer patients with cancer cachexia. Cancer 2019;125:4294-302.

7. Advani SM, Advani PG, VonVille HM, et al. Pharmacological management of cachexia in adult cancer patients: a systematic review of clinical trials. BMC Cancer 2018;18:1174.

8. Granda-Cameron C, Lynch MP. Clinical framework for quality improvement of cancer cachexia. Asia Pac J Oncol Nurs 2018;5:369-76.

9. Hopkinson J. Psychosocial support in cancer cachexia syndrome: the evidence for supported self-management of eating problems during radiotherapy or chemotherapy treatment. Asia Pac J Oncol Nurs 2018;5:358-68.

10. Kang KS, Huh W, Bang Y, et al. Electroacupuncture for chemotherapy-induced anorexia through humoral appetite regulation: A preliminary experimental study. Exp Ther Med 2019;17:2587-97.

11. Jeon JH, Yoon J, Cho CK, et al. Effect of acupuncture for radioactive-iodine-induced anorexia in thyroid cancer patients: a randomized, double-blinded, sham-controlled pilot study. Integr Cancer Ther 2015;14:221-30.

12. Costa RGF, Caro PL, de Matos-Neto EM, et al. Cancer cachexia induces morphological and inflammatory changes in the intestinal mucosa. J Cachexia Sarcopenia Muscle 2019;10:1116-27.

13. Yoon SL, Grundmann O, Williams JJ, et al. Novel intervention with acupuncture for anorexia and cachexia in patients with gastrointestinal tract cancers: a feasibility study. Oncol Nurs Forum 2015;42:E102-9.

14. Wirz-Ridolfi A. The history of ear acupuncture and ear cartography: why precise mapping of auricular points is important. Med Acupunct 2019;31:145-56.

15. Miao J, Liu X, Wu C, et al. Effects of acupressure on chemotherapy-induced nausea and vomiting-a systematic review with meta-analyses and trial sequential analysis of randomized controlled trials. Int J Nurs Stud
2017;70:27-37.

16. Hedlund S, Landgren K. Creating an opportunity to reflect: ear acupuncture in anorexia nervosa - inpatients' experiences. Issues Ment Health Nurs 2017;38:549-56.

17. Kruizenga HM, Seidell JC, de Vet HC, et al. Development and validation of a hospital screening tool for malnutrition: the short nutritional assessment questionnaire (SNAQ). Clin Nutr 2005;24:75-82.

18. Mao JJ, Armstrong K, Farrar JT, et al. Acupuncture expectancy scale: development and preliminary validation in China. Explore (NY) 2007;3:372-7.

19. Eghbali M, Yekaninejad MS, Varaei S, et al. The effect of auricular acupressure on nausea and vomiting caused by chemotherapy among breast cancer patients. Complement Ther Clin Pract 2016;24:189-94.

20. Tang WR, Chen WJ, Yu CT, et al. Effects of acupressure on fatigue of lung cancer patients undergoing chemotherapy: an experimental pilot study. Complement Ther Med 2014;22:581-91.

21. Mao JJ, Bowman MA, Xie SX, et al. Electroacupuncture versus gabapentin for hot flashes among breast cancer survivors: a randomized placebo-controlled trial. J Clin Oncol 2015;33:3615-20.

22. Li FJ, Yang YF, He B. Effect and mechanism of auricular acupuncture on appetite in the rats AOM induced rectal cancer. World Journal of Integrated Traditional and Western Medicine 2018;13:1381-84.

23. Schorr M, Miller KK. The endocrine manifestations of anorexia nervosa: mechanisms and management. Nat Rev Endocrinol 2017;13:174-86.

24. Suo L, Chang X, Zhao Y. The Orexin-A-Regulated Akt/ mTOR pathway promotes cell proliferation through inhibiting apoptosis in pancreatic cancer cells. Front Endocrinol (Lausanne) 2018;9:647.

25. Zhou T, Yang K, Thapa S, et al. Differences in symptom burden among cancer patients with different stages of cachexia. J Pain Symptom Manage 2017;53:919-26.
Cite this article as: Sun L, Mao JJ, Liu Q, Yang Y, He B. Effects of auricular acupuncture on appetite in patients with advanced cancer: a pilot randomized controlled trial. Ann Palliat Med 2020;9(4):1804-1811. doi: 10.21037/apm.2020.04.24 